\title{
ON STABILITY OF FRUSTRATED QUANTUM MAGNETIC STATES IN FULLERENES
}

\author{
A. Koper and M. MUCha
}

Institute of Physics, A. Mickiewicz University, Matejki 48/49, 60-769 Poznań, Poland

Gutzwiller variational ground states $\left|\Phi^{\mathrm{G}}\right\rangle$ of $\pi$ electrons, described by single-band half-filling Hubbard model, were determined for the molecules $\mathrm{C}_{60}$ and $\mathrm{C}_{70}$ and their energies and magnetic properties investigated. To construct these states two types of trial functions were used: generalized spin-density wave $\left|\Phi_{\mathrm{SD}}\right\rangle$ and tight-binding wave $\left|\Phi_{\mathrm{TB}}\right\rangle$. Our results evidently show that the Gutzwiller state $\left|\Phi_{T B}^{G}\right\rangle$ determined by means of function $\left|\Phi_{T B}\right\rangle$ has lower energy than the other investigated variational states $\left|\Phi_{S \mathrm{D}}^{\mathrm{G}}\right\rangle$. Mean values of the operators in the Gutzwiller states were calculated using Monte Carlo method.

PACS numbers: $74.70 . \mathrm{Wz}, 61.46 .+\mathrm{w}, 71.10 .-\mathrm{w}, 71.27 .+\mathrm{a}, 75.10 . \mathrm{Jm}, 02.50 . \mathrm{Ng}$, 02.70.Lq

\section{Introduction}

It is commonly known that, because of presence of pentagons in fullerenes the magnetic ground state of $\pi$ electrons (taken in strong Coulomb repulsion approximation) is frustrated and nontrivial $[1,2]$. On the other hand, it is known that hopping integral $t$ and Coulomb repulsion $U$ are of the same order in the molecules $\mathrm{C}_{N}$. Exact value of these parameters is unknown, but the intermediate values $U / t \approx 3-5$ in IIubbard Hamiltonian may be realistic.

Thus, the problem of stability of frustrated magnetic states in the latter case deserves a closer investigation. We investigate this problem assuming that variational Gutzwiller state is a good approximation of the ground state of the $\mathrm{C}_{N}$ molecules. It is defined as $\left|\Phi^{\mathrm{G}}\right\rangle=\prod_{i}\left(1-g_{i} n_{i \uparrow} n_{i \downarrow}\right)|\Phi\rangle$, where $|\Phi\rangle$ is a trial state, $n_{i \sigma}=c_{i \sigma}^{\dagger} c_{i \sigma}$ and $c_{i \sigma}^{\dagger}, c_{i \sigma}$ are the fermion operators, index $i$ runs over all carbon atoms in $\mathrm{C}_{N}$ and $\sigma$ is a spin label. Variational parameters $g_{i}=g, i=1, \ldots, N$ are determined from minimum of total energy $E(g)=\left\langle\Phi^{\mathrm{G}}|H| \Phi^{\mathrm{G}}\right\rangle /\left\|\Phi^{\mathrm{G}}\right\|^{2}$, where

$$
H=\sum_{\langle i, j\rangle, \sigma, \sigma^{\prime}} t_{i \sigma, j \sigma^{\prime}} c_{i \sigma^{\prime}}^{\dagger} c_{j \sigma^{\prime}}+U \sum_{i} n_{i \uparrow} n_{i \downarrow}
$$

is the $\mathrm{C}_{N}$ molecule's Hubbard Hamiltonian. 


\section{Determination of the electronic ground states}

We chose $N$-electron (half-filling) product state (Slater state) of the lowest energy, obtained from single-particle states of kinetic energy $T=\sum_{\langle i, j\rangle, \sigma, \sigma^{\prime}} t_{i \sigma, j \sigma^{\prime}} \times$ $c_{i \sigma}^{\dagger} c_{j \sigma^{\prime}}$ as our trial state. We consider two types of those states:

(i) Tight-binding (TB) state $\left|\Phi_{\mathrm{TB}}\right\rangle$, when $t_{i \sigma, j \sigma^{\prime}}=t_{i, j} \delta_{\sigma, \sigma^{\prime}}, t_{i, j}$ is different from zero and equal to $t$ only for the nearest neighbors $i, j$ in $\mathrm{C}_{N}$ molecule.

(ii) Generalized spin-density-wave (SD) state $\left|\Phi_{\mathrm{SD}}\right\rangle$, when the kinetic interactions $t_{i \sigma, j \sigma^{\prime}}$ are chosen in agreement with local spin quantization axes, imposed by magnetic ground state of the $\mathrm{C}_{N}$ molecule.

This magnetic state can be obtained from energy minimum of classical IIeisenberg IIamiltonian (cf. [1, 2]), which corresponds to the situation where in IIamiltonian (1) $\left(t_{i \sigma, j \sigma^{\prime}}=t_{i, j} \delta_{\sigma, \sigma^{\prime}}\right)$ we first take the limit $U \rightarrow \infty$ and then the classical limit. Directions of local axes are imposed on each $i$-th carbon atom by equilibrium oricntation of $i$-th spin i.e. by two spherical angles $\theta_{i}$ and $\phi_{i}$. It can be shown that $t_{i \sigma, j \sigma^{\prime}}=t_{i j}\left(R_{i}^{\dagger} R_{j}\right)_{\sigma \sigma^{\prime}}$, where $R_{i}$ is a $\mathrm{SU}(2)$ matrix which rotates initial axes of quantization (corresponding to IIamiltonian TB) in the direction imposed by angles $\theta_{i}$ and $\phi_{i}$.

There are three isomers of the $\mathrm{C}_{60}$ molecule with symmetry groups $D_{2 h}$ (or $T_{h}$ ), $D_{3 d}$ and $D_{5 d}$. Number of differcnt magnetic ground states for the isomers is equal respectively to $2,3,2$ [3]. Since the differences between those states are very small we take for our calculations two states $\left|\Phi_{\mathrm{SD}}^{1}\right\rangle$ and $\left|\Phi_{\mathrm{SD}}^{2}\right\rangle$ corresponding to magnetic valleys of $D_{2 h}$ (or $T_{h}$ ) isomer. The $\mathrm{C}_{70}$ molecule has one isomer, which can be in 3 different ground states corresponding to thrce different states of SD IIamiltonian (1).

In our investigation of the states $\left|\Phi_{\mathrm{TB}}^{\mathrm{G}}\right\rangle$ and $\left|\Phi_{\mathrm{SD}}^{\mathrm{G}}\right\rangle$ properties we calculated functions $E(g)$ as well as mean values of other operators like $M_{z}=n_{i \uparrow}, M_{z z}^{++}=$ $n_{i \uparrow} n_{j \uparrow}, M_{z z}^{+-}=n_{i \uparrow} n_{j \downarrow}$, where the indices $i, j$ belong to pentagon within $\mathrm{C}_{N}$ molecule and are the nearest neighbors.

We proceeded as follows. First we calculated functions $E(g)$, in the interval $0 \leq g \leq 1$ for chosen ratio $U / t$ and state $\left|\Phi^{\mathrm{G}}\right\rangle$ - the minimum of that function corresponds to variational parameter $g^{*}$. Then in the state $\left|\Phi^{\mathrm{G}}\left(g^{*}\right)\right\rangle$ we calculated the energy $E\left(g^{*}\right)$ and mean values of the operators $M_{z}, M_{z z}^{++}, M_{z z}^{+-}$. All the mean values in the Gutzwiller state were calculated using the Monte Carlo method described in [4].

\section{Results}

The results of calculations for the molecule $\mathrm{C}_{60}$ are shown in Figs. 1 and 2. Figure 1 shows that variational state $\left|\Phi_{T B}^{G}\right\rangle$ has clearly lower energy than the $\left|\Phi_{\mathrm{SD}}^{\mathrm{G}, 1}\right\rangle$ and $\left|\Phi_{\mathrm{SD}}^{\mathrm{G}, 2}\right\rangle$ states. This is exactly the opposite to the result obtained by [5] in analogous calculations carried out for IIartrce-Fock (IIF) approximation: energy of the state $\left|\Phi_{\mathrm{TB}}^{\mathrm{HF}}\right\rangle$ is higher (starting from a critical value of parameter $\left.(U / t)_{\mathrm{c}} \approx 2.7\right)$ than the energies of $\left|\Phi_{\mathrm{SD}}^{\mathrm{HF}, \alpha}\right\rangle, \alpha=1,2$ states. We think that this can be explained by the fact that absolute value of correlation energy is lowered in the $\left|\Phi_{\mathrm{TB}}^{\mathrm{HF}}\right\rangle$ state while in the $\left|\Phi_{\mathrm{SD}}^{\mathrm{HF}, \alpha}\right\rangle$ states it is partly correctly taken into account. 
The variational Gutzwiller state seems to be better suited for the description of short-range electron correlations in $\mathrm{C}_{60}$ molecule. Figure 2 shows that in energet-

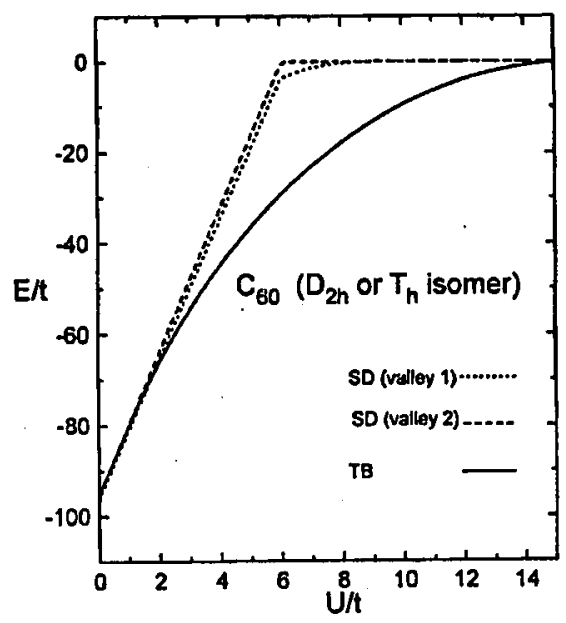

Fig. 1. Dependence of $\mathrm{C}_{60}$ molecule electron ground state energy $E$ (half-filled band) in the Gutzwiller variational state on the Coulomb repulsion energy $U, t$-hopping integral. Continuous line corresponds to stable state (of the lowest energy) $\left|\Phi_{\mathrm{TB}}^{\mathrm{G}}\right\rangle$. The two remaining lines correspond to unstable variational states $\left|\Phi_{\mathrm{SD}}^{\mathrm{G}, \alpha}\right\rangle, \alpha=1,2$.

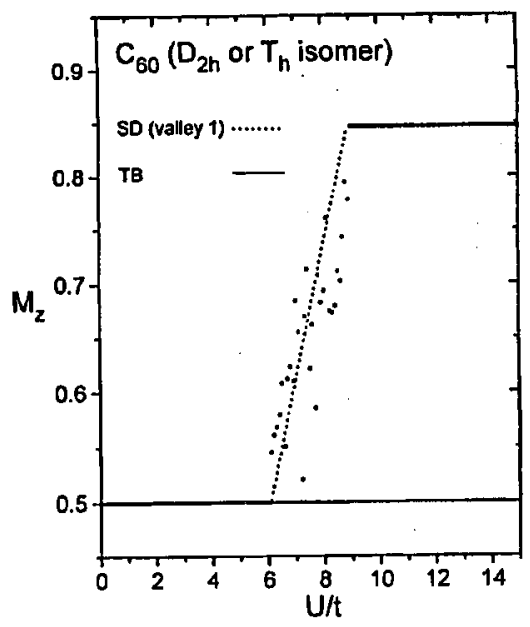

Fig. 2. Mean value $M_{z}=\left\langle n_{i \uparrow}\right\rangle_{\mathrm{G}}$ in the Gutzwiller variational ground state of the $\mathrm{C}_{60}$ molecule as a function $U / t$. The horizontal continuous line $M_{z}=0.5$ corresponds to stable state $\left|\Phi_{\mathrm{TB}}^{\mathrm{G}}\right\rangle$. The stair-like line corresponds to unstable variational state $\left|\Phi_{\mathrm{SD}}^{\mathrm{G}, 1}\right\rangle$. Square dots denote Monte Carlo calculations results, dashed line denotes line fitting. For analogous graph for unstable state $\left|\Phi_{\mathrm{SD}}^{\mathrm{G}, 2}\right\rangle$ the stair-like line is even more clearly visible. 
ically more stable solution we get $M_{z}=\left\langle n_{i \uparrow}\right\rangle_{\mathrm{G}}=0.5$, which corresponds to the situation when there is no distinguished spin direction on the carbon atoms. This picture shows also that in the less stable states $\left|\Phi_{\mathrm{SD}}^{\mathrm{G}, \alpha}\right\rangle$, starting from a critical value of $(U / t)_{\mathrm{c}}^{\prime} \approx 6.1$ we get $M_{z}>0.5$, this means that the local spin quantization axes are subjected to smaller fluctuations in surrounding of classical directions $\theta_{i}$ and $\phi_{i}$. This result, despite the fact that it is energetically instable, is in agreement with analogous result for the states $\left|\Phi_{\mathrm{SD}}^{\mathrm{HF}, \alpha}\right\rangle$, but in this case $(U / t)_{\mathrm{c}} \approx 2.7$ (cf. [5]).

Antiferromagnetic type of correlations should increase with the growth of the ratio $U / t$ in $\mathrm{C}_{60}$. This is exactly the case. The mean values of operators $M_{z z}^{+-}$and $M_{z z}^{++}$calculated in the state $\left|\Phi_{\mathrm{TB}}^{\mathrm{G}}\right\rangle$ tend respectively to $\approx 0.3$ and $\approx 0.15$. In case of the $\mathrm{C}_{70}$ molecule we obtained the same results as for the $\mathrm{C}_{60}$ i.e. energetically more stable is the $\left|\Phi_{\mathrm{TB}}^{\mathrm{G}}\right\rangle$ state than the $\left|\Phi_{\mathrm{SD}}^{\mathrm{G}}\right\rangle$ states. Critical value of the $(U / t)_{\mathrm{c}}^{\prime}$ is also $\approx 6.1$. We expect that the ground state of all higher neutral (not ionized) fullerenes $\mathrm{C}_{N}$ will have similar properties. In real molecules $U / t \approx 3-5$ therefore the antiferromagnetic correlations in $\mathrm{C}_{N}$ are weak. The $\pi$ electrons behave like weakly antiferromagnetically correlated spin liquid. The properties of ground state of crystal consisting of $\mathrm{C}_{N}$ molecules remain an open question.

This work is supported by the Committee for Scientific Research under grant No. 2 P03B 05709.

\section{References}

[1] D. Coffey, S. Trugman, Phys. Rev. Lett. 69, 176 (1992).

[2] A. Koper, J. Stankowski, M. Thomas, Acta Phys. Pol. A 85, 351 (1994).

[3] A. Koper, M. Mucha, to be published.

[4] A. Koper, M. Mucha, Acta Phys. Pol. A 91, 391 (1997).

[5] L. Bergomi, J.P. Blaizot, Th. Jolicoeur, E. Dagotto, Phys. Rev. B 47, 5539 (1993). 